\title{
Significance of Additional Non-Mass Enhancement in Patients with Breast Cancer on Preoperative 3T Dynamic Contrast Enhanced MRI of the Breast
}

\author{
Yun Hee Cho, Kyu Ran Cho, ${ }^{1, *}$ Eun Kyung Park, ${ }^{1}$ Bo Kyoung Seo, Ok Hee Woo, Sung Bum \\ Cho, and Jeoung Won Bae ${ }^{2}$ \\ ${ }^{1}$ Department of Radiology, College of Medicine, Korea University, Seoul, Korea \\ ${ }^{2}$ Department of Surgery, College of Medicine, Korea University, Seoul, Korea \\ ${ }^{*}$ Corresponding author: Kyu Ran Cho, Department of Radiology, Anam Hospital, College of Medicine, Korea University, Inchon-ro, Seongbuk-gu, Seoul 136-705, Korea. Tel: \\ +82-29205578, Fax: +82-29293796, E-mail: krcho@korea.ac.kr
}

Received 2015 June 27; Revised 2015 August 7; Accepted 2015 August 24

\begin{abstract}
Background: In preoperative assessment of breast cancer, MRI has been shown to identify more additional breast lesions than are detectable using conventional imaging techniques. The characterization of additional lesions is more important than detection for optimal surgical treatment. Additional breast lesions can be included in focus, mass, and non-mass enhancement (NME) on MRI. According to the fifth edition of the breast imaging reporting and data system (BI-RADS $®$ ), which includes several changes in the NME descriptors, few studies to date have evaluated NME in preoperative assessment of breast cancer.

Objectives: We investigated the diagnostic accuracy of BI-RADS descriptors in predicting malignancy for additional NME lesions detected on preoperative 3T dynamic contrast enhanced MRI (DCE-MRI) in patients with newly diagnosed breast cancer.

Patients and Methods: Between January 2008 and December 2012, 88 patients were enrolled in our study, all with NME lesions other than the index cancer on preoperative 3T DCE-MRI and all with accompanying histopathologic examination. The MRI findings were analyzed according to the BI-RADS MRI lexicon. We evaluated the size, distribution, internal enhancement pattern, and location of NME lesions relative to the index cancer (i.e., same quadrant, different quadrant, or contralateral breast).

Results: On histopathologic analysis of the 88 NME lesions, 73 (83\%) were malignant and 15 (17\%) were benign. Lesion size did not differ significantly between malignant and benign lesions $(P=0.410)$. Malignancy was more frequent in linear $(P=0.005)$ and segmental $(\mathrm{P}=0.011)$ distributions, and benignancy was more frequent in focal $(\mathrm{P}=0.004)$ and regional $(\mathrm{P}<0.001)$ NME lesions. The highest positive predictive value (PPV) for malignancy occurred in segmental (96.8\%), linear (95.1\%), clustered ring (100\%), and clumped (92.0\%) enhancement. Asymmetry demonstrated a high positive predictive value of $85.9 \%$. The frequency of malignancy was higher for NME lesions located in the same quadrant with the index cancer $(\mathrm{P}=0.006)$, and benignancy was higher in the contralateral breast $(\mathrm{P}=0.015)$. On multivariate analysis, linear $(\mathrm{P}=0.001)$ and segmental $(\mathrm{P}=0.005)$ distributions were significant predictors of malignancy.

Conclusion: The possibility of malignancy is strongly indicated when additional NME lesions show linear or segmental enhancement on preoperative 3T DCE-MRI in patients with recently diagnosed breast cancer.
\end{abstract}

Keywords: Breast Neoplasms, Diagnosis, Non-Mass Enhancement, Preoperative Care, Magnetic Resonance Imaging

\section{Background}

Women with breast cancer are at increased risk of having additional breast lesions (other than the index cancer) in the same or different quadrants of the ipsilateral and contralateral breasts. Previous studies of mastectomy specimens found that 20 - $63 \%$ of additional malignant lesions occurred in the breast ipsilateral to the index cancer and $10 \%$ occurred in the contralateral breast (1-7). The presence of multiple cancer sites may significantly alter the course of treatment; therefore, it is essential to detect and evaluate additional lesions preoperatively.

Magnetic resonance imaging (MRI) is now widely used in preoperative assessment due to its high sensitivity. In particular, 3T dynamic contrast enhanced (DCE) MRI has demonstrated greater utility than mammography or ultrasound and has been shown to detect the extent of the index cancer more precisely and to identify more additional breast lesions than are detectable using conventional imaging techniques $(2,3,8,9)$. Previous studies have demonstrated that preoperative MRI detects 6 - 34\% of additional cancer lesions in the ipsilateral breast (6) and $3-5 \%$ in the contralateral breast $(5,7)$.

The characterization of additional lesions is the next crucial step and even more important than detection. The possibility of malignancy of additional lesions in different quadrants or in the contralateral breast should be evaluated preoperatively, because the existence of additional cancerous lesions might require more extensive excision or preclude breast conserving surgery. Ad-

Copyright ( ) 2016, Tehran University of Medical Sciences and Iranian Society of Radiology. This is an open-access article distributed under the terms of the Creative Commons Attribution-NonCommercial 4.0 International License (http://creativecommons.org/licenses/by-nc/4.0/) which permits copy and redistribute the material just in noncommercial usages, provided the original work is properly cited. 
ditional breast lesions can be seen as a focus, mass, and non-mass enhancement (NME) on MRI, according to the breast imaging reporting and data system (BI-RADS®) lexicon (10). Previous studies have suggested that the combination of particular BI-RADS lexicon descriptors can be useful in determining the likelihood of malignancy for masses, but the MRI characteristics of malignant versus benign NME breast lesions have been debatable (4,11-24). Furthermore, the American college of radiology (ACR) recently published the fifth edition of the BI-RADS lexicon for MRI that institutes several changes in the NME category, including the addition of new descriptors, modification of others, and deletion of descriptors that were rarely helpful clinically (10). To date, there are few studies examining NME according to the new BI-RADS lexicon.

\section{Objectives}

This study was designed to investigate significant predictors of malignancy for NME in lesions other than the index cancer and detected on preoperative 3T DCE-MRI in patients with newly diagnosed breast cancer, based on the new BI-RADS lexicon.

\section{Patients and Methods}

\subsection{Patients and Lesions}

This retrospective study was approved by the institutional review board, and informed consent was waived. We searched the institutional database for cases of breast MRI performed for preoperative staging in patients with newly diagnosed breast cancer from January 2008 to December 2012. The inclusion criteria were as follows: 1) patients with NME lesions other than the index cancer; 2) patients with histopathologically proven lesions; and 3) patients for whom MRI was indicated for preoperative staging prior to neoadjuvant chemotherapy and surgery. Among 322 patients identified from our database search, we excluded 49 patients whose index cancer comprised NME lesions, 117 whose pathology findings were unavailable, and 68 who underwent MRI for purposes other than above inclusion criteria (3). The 117 patients whose pathologic findings did not directly correlate with imaging features were excluded because surgeons did partial mastectomy or wide excision owing to additional NME but without preoperative wire localization. The remaining 88 patients (mean age, 52 years; range, 31 - 75 years) with histopathologically confirmed NME lesions other than the index cancer were included in the study. There was no significant difference in age distribution between the benign and malignant groups. All patients had one NME lesion each. The index cancers were diagnosed as invasive ductal carcinoma (n $=77)$, ductal carcinoma in situ $(\mathrm{n}=8)$, and invasive lobular carcinoma $(\mathrm{n}=3)$.

\subsection{MRI Acquisition}

Breast MRI was performed using a 3.0 T-MRI system (Achieva 3.0 T TX; Philips Healthcare, Best, the Netherlands) or a different 3.0 T-MRI system (Trio Tim; Siemens, Erlangen, Germany) both with a dedicated phased-array breast coil. All imaging were performed with the subjects lying in the prone position. The two different MR systems were assigned to each patient according the MRI suite schedule. T1- and T2-weighted fast spin-echo transverse images were obtained for the two systems. The following image parameters were used for the Achieva system: flip angle, $90^{\circ}$; image matrix, $620 \times 309$; field of view, 581 $\times 342 \mathrm{~mm}$; section thickness, $3 \mathrm{~mm}$; section gap, $0 \mathrm{~mm}$. The following image parameters were used for the Trio Tim system: flip angle, $90^{\circ}$; image matrix, $512 \times 356$; field of view, $360 \times 360 \mathrm{~mm}$; section thickness, $3 \mathrm{~mm}$; section gap, $0.06 \mathrm{~mm}$. A three-dimensional T1-weighted fast spoiled gradient-echo sequence was also performed with transverse imaging using one pre-contrast and six postcontrast dynamic series, immediately following contrast injection and then again after 60, 120, 180, 240, and 300 seconds. The image parameters for the Achieva system were as follows: flip angle, $0^{\circ}$; image matrix, $436 \times 436$; field of view, $330 \times 340 \mathrm{~mm}$; section thickness, $3 \mathrm{~mm}$; section gap, $1.5 \mathrm{~mm}$. The image parameters for the Trio Tim system were as follows: flip angle, $0^{\circ}$; image matrix, 512 $\times 307$; field of view, $360 \times 360 \mathrm{~mm}$; section thickness, 2 $\mathrm{mm}$; section gap, $0 \mathrm{~mm}$. Gadoterate meglumine (Dotarem; Guerbet, Villepinte, France) of $0.1 \mathrm{~mL}$ per kilogram of body weight was injected into an antecubital vein with a power injector (Spectris; Medrad, Indianola, PA, USA) at a rate of $2 \mathrm{~mL} / \mathrm{s}$. For DCE-MRI, post-processing was performed and included early subtraction of pre-contrast images from the first post-contrast images, calculation of time-intensity curves of enhancing regions, and generation of maximum intensity projection images.

\subsection{Image Analysis}

All 3T DCE-MR images were reviewed retrospectively for NME by two experienced breast radiologists (Y.H.C. and K.R.C., with 3 and 14 years' experience, respectively). The readers were both aware that the images were of breast cancer patients, but no information on patients' clinical details or pathologic diagnoses were provided.

The morphologic characteristics of NME were interpreted according to the 2013 ACR BI-RADS® MRI lexicon as follows. NME distribution was classified as either focal, linear, segmental, or regional, and the internal enhancement pattern as homogeneous, heterogeneous, clumped, or clustered ring. The size of the lesion was determined by measuring the largest diameter of the lesion on MIP images, and the lesion was described as symmetrical or asymmetrical. The relative location between the index cancer and NME was also evaluated and then classified into three categories: same quadrant of the ipsilateral breast, different quadrant of the ipsilateral breast, and 
contralateral breast. Each lesion was categorized according to the BI-RADS assessment categories. A consensus interpretation was reached in cases of disagreement.

\subsection{Histopathologic Examination}

The histopathologic examination served as the reference standard. The histological diagnosis was established using a surgically excised sample, and all NME lesions were histopathologically correlated. In 32 patients who underwent mastectomy, those specimens were serially sectioned and correlated with the lesion by referring to the size and location noted on MRI reports. In the remaining 56 patients, all of whom underwent either breast-conserving surgery or excisional biopsy, ultrasound- or mammography-guided wire localization was performed on the additional NME lesions. All diagnoses were made by two experienced breast pathologists in consensus.

\subsection{Statistical Analysis}

The difference in frequency of each MRI feature between benign and malignant lesions was determined using the Fisher's exact test or chi-square test. The sensitivity, specificity, positive predictive value (PPV), and negative predictive value (NPV) were calculated for each MRI feature. Multiple binary logistic regression models were used to identify significant MRI features that differentiated between benign and malignant lesions. The odds ratios with 95\% confidence intervals (CIs) and p-values were estimated for each MR feature. Statistical analyses were performed using SPSS (IBM Corp., Released 2011. IBM SPSS Statistics for Windows, Version 20.0. Armonk, NY: IBM Corp). A p value of less than 0.05 was considered a statistically significant difference.

\section{Results}

\subsection{Histopathological Results}

The histopathologic analysis of the 88 NME lesions revealed 73 (83\%) malignant and 15 (17\%) benign lesions. The 73 malignant NME lesions were identified as follows: ductal carcinoma in situ (DCIS) $(\mathrm{n}=70)$, invasive lobular carcinoma (ILC) $(\mathrm{n}=2)$, and lobular carcinoma in situ (LCIS) $(\mathrm{n}=1)$. The 15 benign NME lesions were diagnosed as follows: atypical ductal hyperplasia $(n=3)$, fibrocystic change $(n=3)$, intraductal papilloma $(n=1)$, and unspecified benign lesions or benign breast tissue $(n=8)$.

\subsection{MRI Findings}

Table 1 summarizes the characteristics of the NME lesions, including patient age, lesion size, and MRI features. Although the maximum diameter of malignant lesions ( $3.7 \mathrm{~cm} \pm 1.6$; range, $0.9-8.8 \mathrm{~cm}$ ) was slightly greater than that of the benign lesions (3.3 cm \pm 1.5 ; range, 1.1 - 6.6 $\mathrm{cm})$, there was no significant difference between the two groups $(\mathrm{P}=0.410)$.

\begin{tabular}{|c|c|c|c|}
\hline & Benign & Malignant & P Value \\
\hline No. of Patients, \% & $15(17.0)$ & $73(83.0)$ & \\
\hline Age, $y^{a}$ & $53.7 \pm 12.1$ & $51.6 \pm 9.6$ & 0.462 \\
\hline Size, $\mathrm{cm}^{\mathrm{a}}$ & $3.3 \pm 1.5$ & $3.7 \pm 1.6$ & 0.410 \\
\hline Distribution, \% & & & 0.034 \\
\hline Focal & $3(20.0)$ & 0 & 0.004 \\
\hline Linear & $2(13.3)$ & $39(53.4)$ & 0.005 \\
\hline Segmental & $1(6.7)$ & $30(41.1)$ & 0.011 \\
\hline Regional & $9(60.0)$ & $4(5.5)$ & $<0.001$ \\
\hline Enhancement, \% & & & $<0.001$ \\
\hline Homogeneous & $4(26.7)$ & $10(13.7)$ & 0.247 \\
\hline Heterogeneous & $9(60.0)$ & $33(45.2)$ & 0.296 \\
\hline Clumped & $2(13.3)$ & $23(31.5)$ & 0.215 \\
\hline Clustered ring & 0 & $7(9.6)$ & 0.598 \\
\hline Symmetricity, \% & & & 0.004 \\
\hline Symmetric & $3(20.0)$ & 0 & \\
\hline Asymmetric & $12(80.0)$ & $73(100)$ & \\
\hline \multicolumn{4}{|l|}{ Location, \% } \\
\hline Same quadrant & $10(66.7)$ & $69(94.5)$ & 0.006 \\
\hline Different quadrant & $2(13.3)$ & $3(4.1)$ & 0.200 \\
\hline Contralateral & $3(20.0)$ & $1(1.4)$ & 0.015 \\
\hline
\end{tabular}

${ }^{\mathrm{a}}$ Data are presented as mean \pm SD. 
Cho YH et al.

The most prevalent features of the benign lesions were a regional distribution (60.0\%) and heterogeneous pattern (60.0\%), and the most prevalent features of the malignant lesions were a linear distribution (53.4\%; Figure 1), heterogeneous pattern (45.2\%), segmental distribution (41.1\%), and clumped pattern (31.5\%; Figure 2). The frequency of malignancy was significantly higher for lesions with a linear
$(\mathrm{P}=0.005)$ and segmental $(\mathrm{P}=0.011)$ distribution, whereas the frequency of benign behavior was significantly higher in lesions with focal $(\mathrm{P}=0.004)$ and regional $(\mathrm{P}<0.001)$ distribution. Although all NME lesions with a clustered ring pattern (Figure 3) were malignant, the distribution of NME with an internal enhancement pattern did not differ significantly between benign and malignant lesions (Table 1).

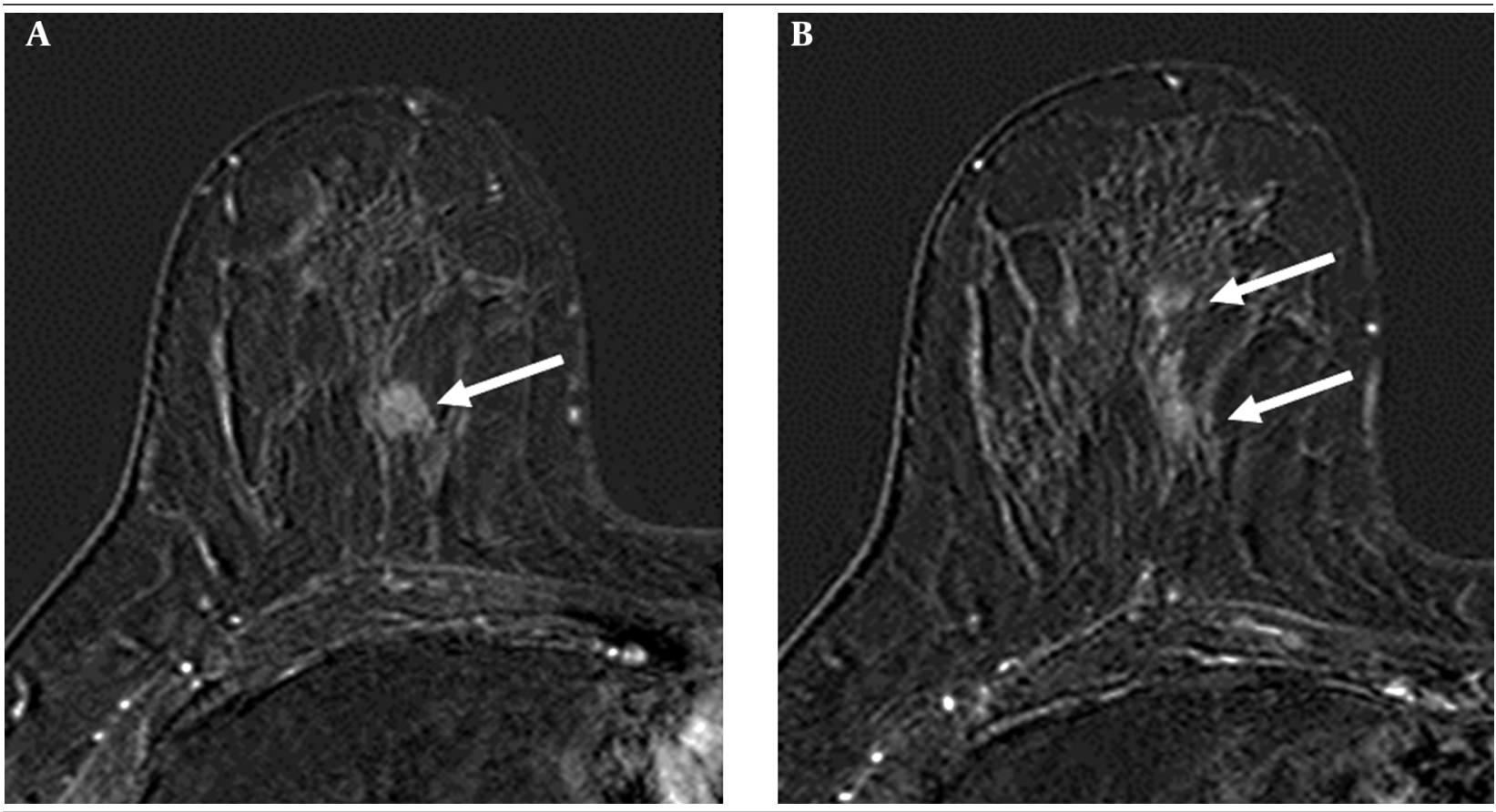

Figure 1. A 52-year-old woman with A, invasive ductal carcinoma in the upper inner quadrant of right breast (arrow); B, The axial post-contrast subtracted image shows heterogeneous linear enhancement (arrows) in the same quadrant as the index cancer. The surgical biopsy revealed ductal carcinoma in situ.
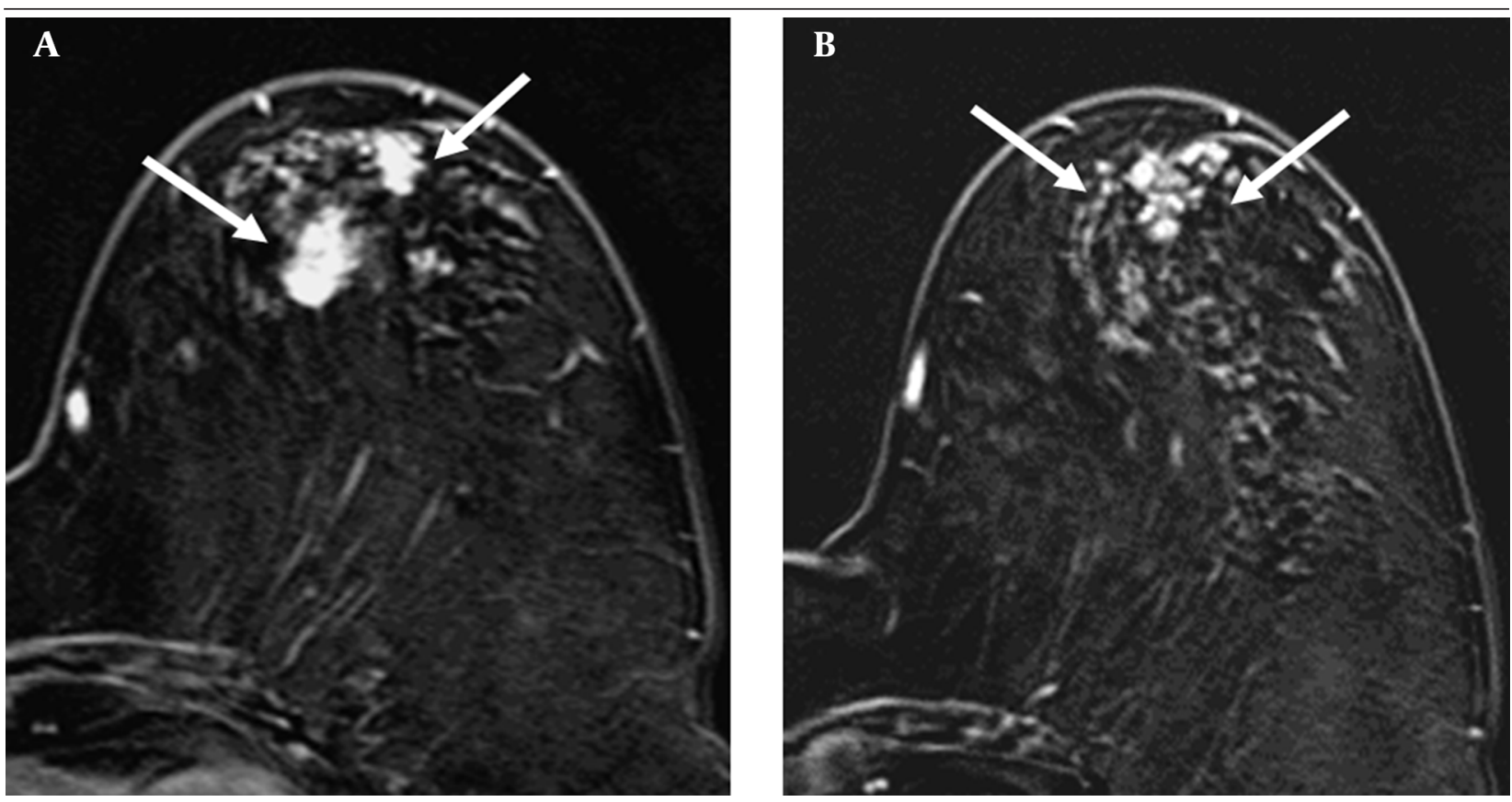

Figure 2. A 69-year-old woman with A, invasive ductal carcinoma in the upper outer quadrant of left breast (arrows); B, The axial post-contrast subtracted image depicts segmental clumped enhancement (arrows) in the same quadrant as the index cancer. The surgical biopsy revealed ductal carcinoma in situ. 
Cho YH et al.

Table 2 lists the sensitivity, specificity, PPV, and NPV according to the NME distribution and internal enhancement pattern. The distribution patterns with high specificity were linear (86.7\%) and segmental (93.3\%) distributions, and the internal enhancement patterns with high specificity were clumped (92.0\%) and clustered ring (100.0\%) enhancement. The features with high PPV for malignancy were segmental distribution $(96.8 \%, \mathrm{P}=0.011)$ and clustered ring enhancement $(100.0 \%, \mathrm{P}=0.598)$.

Symmetric lesions were found in three patients, and all three lesions were diagnosed as benign (Table 1). The presence or absence of symmetry differed significantly between malignant and benign lesions $(\mathrm{P}=0.004)$. Asymmetry demonstrated a high PPV for malignancy of $85.9 \%$ (Table 2).

NME lesions were found most frequently in the same quadrant of the ipsilateral breast as the index cancer (Table 1). NME lesions located in the same quadrant as the index cancer were significantly more likely to be malignant $(\mathrm{P}=0.006)$, and NME lesions in the contralateral breast were more likely benign $(\mathrm{P}=0.015)$.

Of 88 NME lesions, $6.8 \%$ had a BI-RADS category 3 assessment, $62.5 \%$ had a BI-RADS category 4 assessment, and $30.7 \%$ had a BI-RADS category 5 assessment on the basis of MRI findings. Of the six lesions with a BI-RADS category 3 assessment, five were proven benign and one was malignant, yielding a PPV of 83.3\% (95\% CI: 36.5-99.1\%). Of the 55 lesions with a BI-RADS category 4 assessment, 45 malignancies were diagnosed, yielding a PPV of $81.8 \%$ (95\% CI: 68.6-90.5\%). Of the 27 lesions with a BI-RADS category 5 assessment, all lesions were proven malignant, yielding a PPV of $100 \%$ (95\% CI: 84.5-100\%).
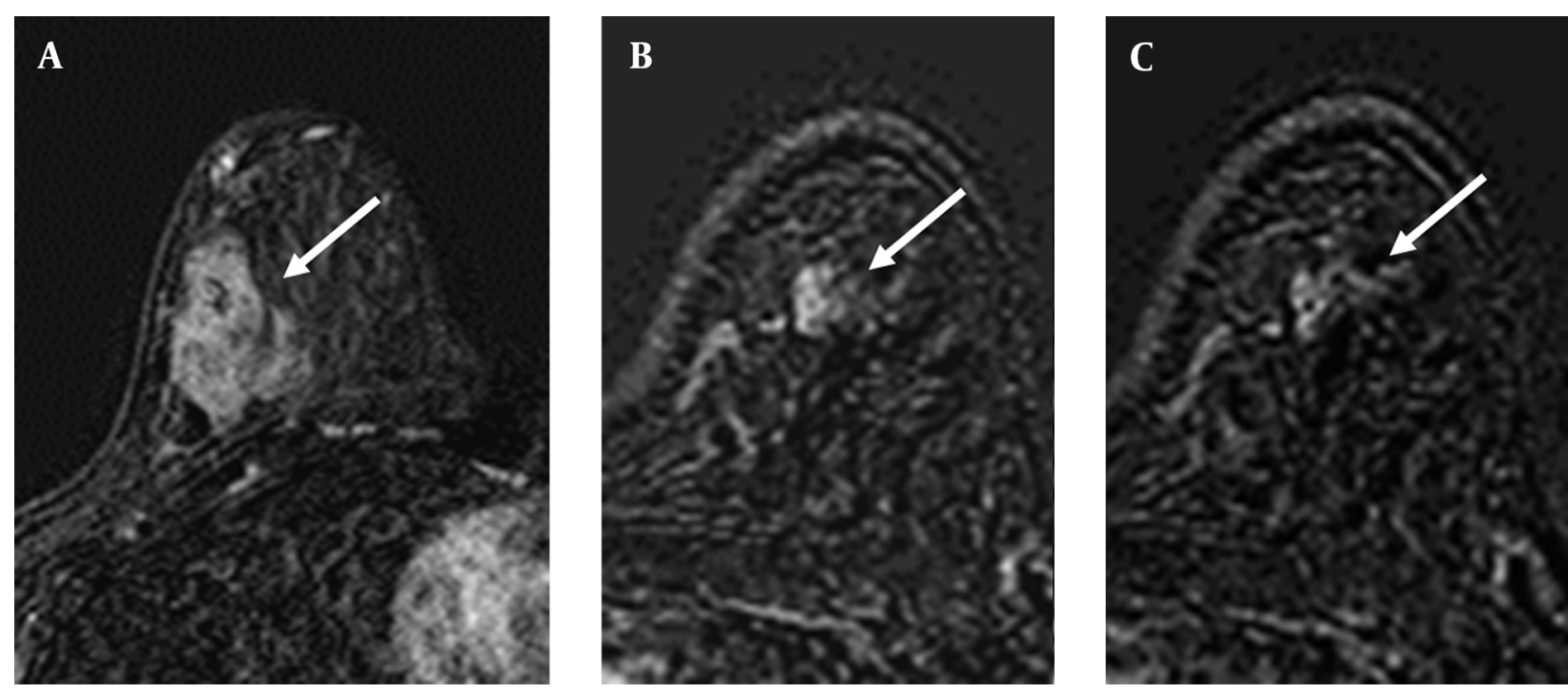

Figure 3. A 47-year-old woman with A, invasive ductal carcinoma in the right breast (arrow); B, C. The axial post-contrast subtracted consecutive images demonstrate focal clustered ring enhancement (arrow) in the contralateral breast. The surgical biopsy revealed ductal carcinoma in situ.

Table 2. Estimated Sensitivity, Specificity, Positive and Negative Predictive Values, and Positive and Negative Likelihood Ratios of MRI Features in Prediction of Malignancy ${ }^{\mathrm{a}}$

\begin{tabular}{lcccccc}
\hline & Sensitivity & Specificity & PPV & NPV & PLR & NLR \\
\hline Focal & $0.0(0.0-6.2)$ & $80.0(51.4-94.7)$ & $0.0(0.0-69.0)$ & $14.1(7.8-23.8)$ & 0 & $1.25(1.21-1.29)$ \\
Linear & $53.4(41.4-65.0)$ & $86.7(58.4-97.7)$ & $95.1(82.2-99.2)$ & $27.7(16.1-42.9)$ & $4.00(1.18-14.82)$ & $0.54(0.41-0.70)$ \\
Segmental & $41.1(29.9-53.2)$ & $93.3(66.0-99.7)$ & $96.8(81.5-99.8)$ & $24.6(14.5-38.0)$ & $6.16(0.91-41.77)$ & $0.63(0.52-0.77)$ \\
Regional & $5.5(1.8-14.2)$ & $40.0(17.5-67.1)$ & $30.8(10.4-61.1)$ & $8.0(3.3-17.2)$ & $0.09(0.03-0.26)$ & $2.36(1.84-3.04)$ \\
Homogeneous & $13.7(7.1-24.2)$ & $73.3(44.8-91.1)$ & $71.4(42.0-90.4)$ & $14.9(9.0-25.5)$ & $0.51(0.19-1.42)$ & $1.18(1.02-1.35)$ \\
Heterogeneous & $45.2(33.7-57.2)$ & $40.0(33.7-57.2)$ & $78.6(62.8-89.2)$ & $13.0(5.4-27.0)$ & $0.75(0.46-1.22)$ & $1.37(0.91-2.05)$ \\
Clumped & $31.5(21.4-43.6)$ & $86.7(58.4-97.7)$ & $92.0(72.5-98.6)$ & $20.6(11.9-33.0)$ & $2.36(0.62-8.97)$ & $0.79(0.66-0.94)$ \\
Clustered ring & $9.6(4.3-19.3)$ & $100.0(74.7-100.0)$ & $100.0(56.1-100.0)$ & $18.5(11.1-29.1)$ & - & $0.90(0.84-0.97)$ \\
Asymmetry & $100.0(93.8-100.0)$ & $20.0(5.3-48.6)$ & $85.9(76.2-92.2)$ & $100.0(31.0-100.0)$ & $1.25(0.97-1.61)$ & 0
\end{tabular}

Abbreviations: NLR, negative likelihood ratio; NPV, negative predictive value; PLR, positive likelihood ratio; PPV, positive predictive value.

${ }^{\mathrm{a}}$ Data are presented as percent( $95 \%$ Confidence Interval). 


\subsection{Binary Logistic Regression Analysis}

Of the MR features analyzed in the logistic regression model, linear (odds ratio $=66.4 ; 95 \% \mathrm{CI}, 5.66-779.68 ; \mathrm{P}=$ 0.001 ) and segmental (odds ratio $=35.1 ; 95 \% \mathrm{CI}, 3.01-411.10$; $\mathrm{P}=0.005$ ) patterns were the only significant predictors of malignancy. None of the internal enhancement patterns or locations demonstrated any statistically significant association with benign or malignant lesions.

\section{Discussion}

It is well known that breast cancer can be multifocal or multicentric in the ipsilateral and even in the contralateral breast to the index cancer. As a result, MRI has become an essential imaging tool for preoperative assessment in patients diagnosed with breast cancer. MRI can identify additional cancer foci that would otherwise remain undetected by clinical assessment combined with mammography or ultrasound $(2,3,8,9)$. It is unclear whether the detection of additional malignant foci improves patient outcomes, but identification of these additional lesions can help in the selection of appropriate patients for breast conserving surgery or local excision. In a study conducted by Houssami et al.(3), some patients underwent a more extensive surgery due to MRI results: the conversion rate from a wide local excision to mastectomy was $8.1 \%$, and the conversion rate from a wide local excision to more extensive surgery (such as an additional or wider excision) was $11.3 \%$.

It is common to find additional lesions in the form of NME on preoperative MRI. Liberman et al. (6) found that NME lesions comprised 32\% of additional lesions in the ipsilateral breast, and in another study, the same investigators reported that NME comprised 33\% of the additional lesions in the contralateral breast (7). The malignant potential of additional NME lesions should be elucidated because their malignant behavior can alter the course of treatment. Although several studies have characterized the MRI features of NME in differentiating malignant and benign lesions (6, 7, 11-17, 19-25), standardized interpretative criteria based on the BI-RADS MRI lexicon for NME do not exist. Our study focused on additional NME lesions identified during preoperative MRI, a scenario occasionally encountered in the clinical setting.

Our findings demonstrated that linear and segmental distribution patterns were the strongest indicators of malignancy. Linear and segmental distribution patterns were also among the most frequent morphologic findings in malignant lesions and showed high PPVs for malignancy of $95.1 \%$ and $96.8 \%$, respectively.

Our PPV for the linear distribution pattern is much higher than rates in previously published data. Liberman et al.(13) reported that the overall PPV for linear- and branching-ductal patterns was $26 \%$. Morakkabati-Spitz et al. (24) found a 34\% PPV for linear and segmental enhancement. Wilhelm et al. (22) reported a 39.7\% and 36.7\% PPV for linear and ductal enhancement, respectively. Because "ductal" enhancement is reclassified as "linear" in the updated BI-RADS lexicon, we surmise that the previous PPV for "ductal" enhancement contributes to the high PPV for "linear" enhancement in the current data.

Clustered ring enhancement was added as a descriptor in the 2013 BI-RADS lexicon for MRI. In our study, all NME lesions showing clustered ring enhancement were diagnosed as malignant, which is consistent with findings by Tozaki et al. (20). However, the frequency of cluster ring enhancement differed significantly between benign and malignant lesions, and was not a significant predictor of malignancy in our study. This likely reflects the considerable size difference between benign and malignant lesions, which is discussed in the study limitations below.

Asymmetry was associated with an $85.9 \%$ likelihood of malignancy. This is comparable to the $75 \%$ PPV reported by Wilhelm et al. (22) and was unsurprising since asymmetry is usually considered a feature highly suspicious of malignancy.

In this study, the malignant rate of NME lesions was considerably high. Since we focused on additional NME lesions identified on preoperative imaging in newly diagnosed breast cancer, and since breast cancer can be multifocal and multicentric, we expected our malignancy rate to be higher than rates in other NME studies that include MRI performed for screening or other clinical purposes. Also, 95.9\% of malignant lesions were confirmed as DCIS, which mirrors the fact that DCIS is commonly present in conjunction with invasive ductal carcinoma and that it frequently appears as NME.

We should note a few limitations of our study. First, it was conducted retrospectively, and second, the size difference between malignant and benign lesions was large and may have caused statistical selection bias. Besides the reasons mentioned above, most of the additional lesions considered benign were not pathologically diagnosed (though most of them were stable or disappeared on follow-up breast MRI). Third, we did not evaluate the kinetic features of additional NME lesions in this study. Further studies that include a kinetic analysis may have diagnostic benefit in the evaluation of NME lesions.

In conclusion, malignancy may be suspected when additional NME lesions with linear, segmental, clumped, or clustered ring enhancement and asymmetry are encountered in the same quadrant of the ipsilateral breast to the index cancer on preoperative 3T DCE-MRI. Percutaneous biopsy or wide surgical excision should be considered in these cases to potentially diagnosis multiple occult breast cancers.

\section{Footnotes}

Authors' Contribution:Study concept and design: Bo Kyoung Seo, Kyu Ran Cho; acquisition of data: Yun Hee Cho, Eun Kyung Park, Ok Hee Woo; analysis and interpretation of data: Yun Hee Cho, Kyu Ran Cho; drafting of the manuscript: Yun Hee Cho, Kyu Ran Cho; critical revision 
of the manuscript for important intellectual content: Kyu Ran Cho; statistical analysis: Yun Hee Cho, Ok Hee Woo; administrative, technical, and material support: Bo Kyoung Seo, Jeoung Won Bae; study supervision: Kyu Ran Cho, Sung Bum Cho

Financial Disclosure:The authors have no conflicts of interest to declare.

Funding/Support:This original research is supported by Guerbet Korea.

\section{References}

1. Brennan ME, Houssami N, Lord S, Macaskill P, Irwig L, Dixon JM, et al. Magnetic resonance imaging screening of the contralateral breast in women with newly diagnosed breast cancer: systematic review and meta-analysis of incremental cancer detection and impact on surgical management. J Clin Oncol. 2009;27(33):56409. doi:10.1200/JCO.2008.21.5756. [PubMed:19805685]

2. Girardi V, Carbognin G, Camera L, Baglio I, Bucci A, Bonetti F, et al. Multifocal, multicentric and contralateral breast cancers: breast MR imaging in the preoperative evaluation of patients with newly diagnosed breast cancer. Radiol Med. 2011;116(8):1226-38. doi:10.1007/s11547-011-0704-7. [PubMed: 21744256]

3. Houssami N, Ciatto S, Macaskill P, Lord SJ, Warren RM, Dixon JM, et al. Accuracy and surgical impact of magnetic resonance imaging in breast cancer staging: systematic review and metaanalysis in detection of multifocal and multicentric cancer. $J$ Clin Oncol. 2008;26(19):3248-58. doi: 10.1200/JCO.2007.15.2108. [PubMed:18474876]

4. Lee SG, Orel SG, Woo IJ, Cruz-Jove E, Putt ME, Solin LJ, et al. MR imaging screening of the contralateral breast in patients with newly diagnosed breast cancer: preliminary results. Radiology. 2003;226(3):773-8. doi: 10.1148/radiol.2263020041. [PubMed: 12601182]

5. Lehman CD, Gatsonis C, Kuhl CK, Hendrick RE, Pisano ED, Hanna L, et al. MRI evaluation of the contralateral breast in women with recently diagnosed breast cancer. NEngl J Med.2007;356(13):1295303. doi:10.1056/NEJMoa065447. [PubMed:17392300]

6. Liberman L, Morris EA, Dershaw DD, Abramson AF, Tan LK. MR imaging of the ipsilateral breast in women with percutaneously proven breast cancer. AJR Am J Roentgenol. 2003;180(4):901-10. doi:10.2214/ajr.180.4.1800901. [PubMed: 12646427]

7. Liberman L, Morris EA, Kim CM, Kaplan JB, Abramson AF, Menell $\mathrm{JH}$, et al. MR imaging findings in the contralateral breast of women with recently diagnosed breast cancer. AJR Am J Roentgenol. 2003;180(2):333-41. doi: 10.2214/ajr.180.2.1800333. [PubMed: 12540428]

8. Berg WA, Gutierrez L, NessAiver MS, Carter WB, Bhargavan M, Lewis RS, et al. Diagnostic accuracy of mammography, clinical examination, US, and MR imaging in preoperative assessment of breast cancer. Radiology. 2004;233(3):830-49. doi: 10.1148/radiol.2333031484. [PubMed:15486214]

9. Boetes C, Mus RD, Holland R, Barentsz JO, Strijk SP, Wobbes T, et al. Breast tumors: comparative accuracy of MR imaging relative to mammography and US for demonstrating extent. Radiology. 1995;197(3):743-7. doi: 10.1148/radiology.197.3.7480749. [PubMed: 7480749]

10. American College of Radiology. ACR BI-RADS atlas : breast imaging reporting and data system. Reston, VA; American College of Radiology. 2013.

11. Ballesio L, Di Pastena F, Gigli S, D'Ambrosio I, Aceti A, Pontico $\mathrm{M}$, et al. Non mass-like enhancement categories detected by breast MRI and histological findings. Eur Rev Med Pharmacol Sci.
2014;18(6):910-7. [PubMed: 24706319]

12. Gutierrez RL, DeMartini WB, Eby PR, Kurland BF, Peacock S, Lehman CD. BI-RADS lesion characteristics predict likelihood of malignancy in breast MRI for masses but not for nonmasslike enhancement. AJR Am J Roentgenol. 2009;193(4):994-1000. doi: 10.2214/AJR.08.1983. [PubMed: 19770321]

13. Liberman L, Morris EA, Lee MJ, Kaplan JB, LaTrenta LR, Menell JH, et al. Breast lesions detected on MR imaging: features and positive predictive value. AJR Am J Roentgenol. 2002;179(1):171-8. doi: 10.2214/ajr.179.1.1790171. [PubMed:12076929]

14. Newell D, Nie K, Chen JH, Hsu CC, Yu HJ, Nalcioglu O, et al. Selection of diagnostic features on breast MRI to differentiate between malignant and benign lesions using computer-aided diagnosis: differences in lesions presenting as mass and nonmass-like enhancement. Eur Radiol. 2010;20(4):771-81. doi: 10.1007/s00330-009-1616-y. [PubMed:19789878]

15. Nunes LW, Schnall MD, Orel SG, Hochman MG, Langlotz CP, Reynolds CA, et al. Breast MR imaging: interpretation model. Radiology. 1997;202(3):833-41. doi: 10.1148/radiology.202.3.9051042. [PubMed: 9051042]

16. Nunes LW, Schnall MD, Siegelman ES, Langlotz CP, Orel SG, Sullivan D, et al. Diagnostic performance characteristics of architectural features revealed by high spatial-resolution MR imaging of the breast. AJR Am J Roentgenol. 1997;169(2):409-15. doi: 10.2214/ ajr.169.2.9242744. [PubMed: 9242744]

17. Sakamoto N, Tozaki M, Higa K, Tsunoda Y, Ogawa T, Abe S, et al. Categorization of non-mass-like breast lesions detected by MRI. Breast Cancer. 2008;15(3):241-6. doi: 10.1007/s12282-007-0028-6. [PubMed: 18224381]

18. Schnall MD, Blume J, Bluemke DA, DeAngelis GA, DeBruhl N, Harms S, et al. Diagnostic architectural and dynamic features at breast MR imaging: multicenter study. Radiology. 2006;238(1):4253. doi: 10.1148/radiol.2381042117. [PubMed:16373758]

19. Shao Z, Wang H, Li X, Liu P, Zhang S, Cao S. Morphological distribution and internal enhancement architecture of contrast-enhanced magnetic resonance imaging in the diagnosis of non-mass-like breast lesions: a meta-analysis. Breast J. 2013;19(3):259-68. doi: 10.1111/tbj.12101. [PubMed:23614364]

20. Tozaki M, Fukuda K. High-spatial-resolution MRI of non-masslike breast lesions: interpretation model based on BI-RADS MRI descriptors. AJR Am J Roentgenol. 2006;187(2):330-7. doi: 10.2214/ ajr.187.3.w330. [PubMed:16861534]

21. Uematsu T, Kasami M. High-spatial-resolution 3-T breast MRI of nonmasslike enhancement lesions: an analysis of their features as significant predictors of malignancy. AJR Am J Roentgenol. 2012;198(5):1223-30. doi:10.2214/AJR.11.7350. [PubMed: 22528918]

22. Wilhelm A, McDonough MD, DePeri ER. Malignancy rates of nonmasslike enhancement on breast magnetic resonance imaging using American College of Radiology Breast Imaging Reporting and Data System descriptors. Breast J. 2012;18(6):523-6. doi:10.1111/ tbj.12008. [PubMed: 23009294]

23. Yabuuchi H, Matsuo Y, Kamitani T, Setoguchi T, Okafuji T, Soeda H, et al. Non-mass-like enhancement on contrast-enhanced breast MR imaging: lesion characterization using combination of dynamic contrast-enhanced and diffusion-weighted MR images. Eur J Radiol. 2010;75(1):e126-32. doi: 10.1016/j.ejrad.2009.09.013. [PubMed:19796900]

24. Morakkabati-Spitz N, Leutner C, Schild H, Traeber F, Kuhl C. Diagnostic usefulness of segmental and linear enhancement in dynamic breast MRI. Eur Radiol. 2005;15(9):2010-7. doi: 10.1007| s00330-005-2755-4. [PubMed:15841382]

25. Gity M, Ghazi Moghadam K, Jalali AH, Shakiba M. Association of Different MRI BIRADS Descriptors With Malignancy in Non MassLike Breast Lesions. Iran Red Crescent Med J. 2014;16(12):e26040. doi:10.5812/ircmj.26040. [PubMed:25763248] 